\title{
KULLBACK-LEIBLER ENTROPY ANALYSIS OF THE ELECTROENCEPHALOGRAM BACKGROUND ACTIVITY IN ALZHEIMER'S DISEASE PATIENTS
}

\author{
Daniel Abásolo ${ }^{1}$, Dionisio Muñoz ${ }^{2}$, Pedro Espino ${ }^{3}$ \\ ${ }^{1}$ Centre for Biomedical Engineering, Division of Mechanical, Medical and Aerospace Engineering, Faculty of Engineering \\ and Physical Sciences, University of Surrey, United Kingdom \\ d.abasolo@surrey.ac.uk \\ ${ }^{2}$ E.T.S. Ingenieros de Telecomunicación, Universidad de Valladolid, Campus Miguel Delibes, Paseo Belén 15, 47011, \\ Valladolid, Spain. \\ ${ }^{3}$ Hospital Clínico San Carlos, c/Profesor Martín Lagos s/n, 28040, Madrid, Spain.
}

\begin{abstract}
Alzheimer's disease (AD) is the most frequent form of dementia in western countries. An early detection would be beneficial, but currently diagnostic accuracy is relatively poor. In this study, differences in information content between cortical areas in $12 \mathrm{AD}$ patients and 11 control subjects were assessed with Kullback-Leibler (KL) entropy. KL entropy measures the degree of similarity between two probability distributions. EEGs were recorded from 19 scalp electrodes and KL entropy values of the EEGs in both groups were estimated for the local, distant and interhemispheric electrodes. KL entropy values were lower in $\mathrm{AD}$ patients than in age-matched control subjects, with significant effects for diagnosis and brain region ( $p<0.05$, two-way ANOVA). No significant interaction for diagnosis $\mathrm{X}$ region was found $(p=0.7671)$. Additionally a one-way ANOVA showed that KL entropy values were significantly lower in AD patients $(p<0.05)$ for the distant electrodes on the right hemisphere. These results suggest that KL entropy highlights information content changes in the EEG due to AD. However, further studies are needed to address the possible usefulness of KL entropy in the characterisation and early detection of $\mathrm{AD}$.
\end{abstract}

\section{KEY WORDS}

Biomedical signal processing, Electroencephalogram, Alzheimer's disease, Kullback-Leibler entropy.

\section{Introduction}

Alzheimer's disease (AD) is a primary degenerative dementia of unknown aetiology. It is the main cause of dementia in western countries [1]. AD is characterised by impairments in cognition and memory during several years before the death of the patient. The rise in life expectancy will likely increase its prevalence, since ageing is the greatest known risk factor. Patients with a diagnosis of $\mathrm{AD}$ may wander, be unable to engage in conversation, appear to be non-responsive, become helpless, and need complete care and attention [2]. From a structural point of view, the accumulation of amyloid plaques between nerve cells in the brain and the appearance of neurofibrillary tangles inside nerve cells are considered hallmarks of $\mathrm{AD}$ [3].

An early diagnosis would help to reduce brain damage and support the adoption of more efficient drug taking strategies. Nowadays, clinical diagnosis of $\mathrm{AD}$ involves several kinds of evaluations, such as medical history studies, laboratory tests, physical and neurological evaluation and mental status tests, among others. However, the diagnostic accuracy in $\mathrm{AD}$ is relatively poor and is only definite by necropsy. Hence, new tools are needed to help in this complex task.

The electroencephalogram (EEG), the recording of the brain electrical activity with electrodes, has been extensively used in dementia research. Preliminary studies suggest that the EEG in AD patients shows a slowing (i.e. the power spectrum shifts to lower frequencies), although this change usually does not appear in the early stages of the disease [4]. Furthermore, a decrease of coherence among cortical areas in AD patients' EEGs has also been reported [2].

Conventional EEG analysis relies on visual inspection or on linear methods. However, non-linearity is introduced in the brain at the cellular level [5]. Thus, EEG fluctuations are not best described with linear techniques and it might be possible to characterise the brain electrical activity more appropriately with advanced signal processing techniques.

One possible solution lies in computing the entropy of the EEG. Entropy is a concept addressing randomness and predictability, with greater entropy often associated with more randomness and less system order. Shannon defined the information concept of entropy as the expected value (i.e. the average amount) of the information of a probability distribution [6]. Since this groundbreaking work, Shannon's definition of entropy has been applied, modified and proven valid in a variety of fields. In particular, different metrics derived from information theory have been used in time series analysis, for instance, to quantify the difference between 
probability distributions [7]. One of this metrics is the Kullback-Leibler (KL) entropy [8], [9]. KL entropy measures the degree of similarity between two probability distributions and can be interpreted as a method quantifying differences in information content.

In this pilot study, differences in information content between cortical areas in both $\mathrm{AD}$ and control subjects have been investigated by estimating the KL entropy between EEG electrodes. We wanted to test the hypothesis that the information content between cortical areas in AD patients would be significantly different than in control subjects.

\section{Material and methods}

\subsection{Subjects}

Twenty-three subjects were selected to take part in this pilot study. Twelve patients ( 6 men and 6 women; age $=$ $72.8 \pm 8.0$ years, mean \pm standard deviation, SD) were recruited from the Alzheimer's Patients' Relatives Association of Valladolid, Spain (AFAVA). All of them fulfilled the criteria of probable AD. The mean MiniMental State Examination (MMSE), a quick and simple way to evaluate cognitive function [10], score for the patients was $13.3 \pm 5.6$ (Mean $\pm \mathrm{SD}$ ).

The control group was formed by 11 age-matched control subjects without past or present neurological disorders $(7$ men and 4 women; age $=72.8 \pm 6.1$ years, mean $\pm \mathrm{SD}$ ). The MMSE score was 30 for all controls.

The research protocol was approved by the local ethics committee. All control subjects and all caregivers of the patients gave their informed consent for participation in this study.

\subsection{EEG signals}

EEGs were recorded over 5 minutes from the 19 scalp loci of the international 10-20 system (electrodes F3, F4, F7, F8, Fp1, Fp2, T3, T4, T5, T6, C3, C4, P3, P4, O1, O2, $\mathrm{Fz}, \mathrm{Cz}$ and $\mathrm{Pz}$ ) using a Profile Study Room 2.3.411 EEG equipment (Oxford Instruments) at the University Hospital of Valladolid (Spain). The sampling frequency was $256 \mathrm{~Hz}$ and a 12-bit A-to-D conversion was used to digitise the data. Recordings were made with eyes-closed condition to minimise artefacts. Furthermore, all EEGs were visually inspected by a specialist physician to select 5 second artefact-free epochs (1280 points). Additionally, all recordings were digitally filtered with a band-pass filter with cut-off frequencies at $0.5 \mathrm{~Hz}$ and at $40 \mathrm{~Hz}$ in order to remove residual electromyographic activity.

\subsection{Kullback-Leibler entropy}

Let us consider a discrete random variable with outcomes $x_{k}(k=1, \ldots, n)$ with probabilities $p_{k}$. Shannon's entropy is defined as [6]:

$$
H=-\sum_{k=1}^{n} p_{k} \cdot \ln p_{k}
$$

We can assume that $k$ represents a frequency index and $p_{k}$ is the normalised value of the power spectral density [7], [11].

If we have two different probability distributions $p_{k}$ and $q_{k}$, we can define the KL entropy as follows [8], [9]:

$$
K(p \mid q)=-\sum_{k=1}^{n} p_{k} \cdot \ln \frac{p_{k}}{q_{k}}
$$

KL entropy is positive and only vanishes when $p_{k}$ and $q_{k}$ are equal. It measures how similar are both probability distributions [7].

In this study, $p_{k}$ and $q_{k}$ represent EEG samples from different electrodes but the same epoch. We estimated the KL entropy values between electrodes for seven different brain regions:

- Local anterior on the left hemisphere (electrodes located over frontal and antero-temporal regions for pairs of anterior brain region: Fp1-F7, Fp1F3, Fp1-C3, F7-C3, F3-C3).

- Local anterior on the right hemisphere (Fp2-F8, Fp2-F4, Fp2-C4, F8-C4, F4-C4).

- Local posterior on the left hemisphere (between electrodes located on the temporal, parietal, and occipital regions: O1-P3, O1-T5, O1-C3, P3C3, T5-C3).

- Local posterior on the right hemisphere $(\mathrm{O} 2-\mathrm{P} 4$, O2-T6, O2-C4, P4-C4, T6-C4).

- Distant electrodes on the left hemisphere (O1Fp1, O1-F7, O1-F3, P3-Fp1, P3-F7, P3-F3, T5-Fp1, T5-F7, T5-F3).

- Distant electrodes on the right hemisphere (O2Fp2, O2-F8, O2-F4, P4-Fp2, P4-F8, P4-F4, T6-Fp2, T6-F8, T6-F4).

- Interhemispheric electrodes.

These definitions of local anterior, local posterior, distant and interhemispheric pairs of electrodes have been used before in studies estimating local and distant coherences [12] and cross mutual information [13] of the EEG in AD patients.

\subsection{Statistical analysis}

The Kolmogorov-Smirnov test was used to check the normality of the distributions of the KL entropy values for both groups.

Group differences were analysed with a two way ANOVA, one for diagnosis (patients vs. controls) and one for brain region (7 different regions). Furthermore, a one way ANOVA was used to analyse differences between $\mathrm{KL}$ entropy values from $\mathrm{AD}$ patients and controls for the different electrode combinations. 


\section{Results}

For both groups (patients with a diagnosis of $\mathrm{AD}$ and control subjects) KL entropy values followed a normal distribution.

In general, the EEG of AD patients was characterised by lower KL entropy values than the EEG of control subjects (see Figure 1). A two-way ANOVA yielded significant effects for diagnosis $(p<0.05)$ and region $(p<$ $0.05)$. On the other hand, no significant interaction for diagnosis $\mathrm{X}$ region was found $(p=0.7671)$.

Table 1 summarises the average values for the 7 regions previously defined. As it can be noticed, the differences are more evident between distant electrodes. This indicates that the differences in information content between distant regions were reduced in $\mathrm{AD}$ patients.

Differences between KL entropy values from AD patients and control subjects for the different electrode combinations were evaluated with a one-way ANOVA. KL entropy values were only significantly lower in AD patients $(p<0.05)$ for the combination of distant electrodes located on the right hemisphere $(\mathrm{O} 2-\mathrm{Fp} 2, \mathrm{O} 2-$ F8, O2-F4, P4-Fp2, P4-F8, P4-F4, T6-Fp2, T6-F8, T6F4). Table 2 summarises the $p$-values for these one way ANOVA tests. However, these differences were no longer significant when the Bonferroni correction was used.

\section{Discussion}

The EEG records the brain electrical activity and can be useful in AD diagnosis. Nevertheless, conventional EEG analysis relies on visual inspection or relatively simple signal processing techniques. It might be argued that, due to the complex nature of the electrical brain activity, advanced signal processing techniques could provide information unavailable with conventional techniques and that this could help in AD diagnosis. Thus, in this pilot study we wanted to evaluate if KL entropy analysis of EEG recordings could provide relevant information for

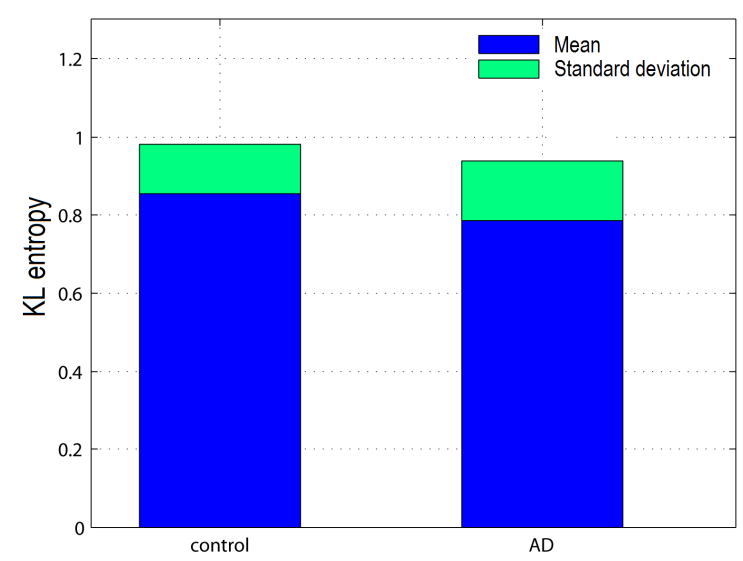

Figure 1. Average KL entropy values for control subjects and $\mathrm{AD}$ patients.
Table 1. Mean and SD of KL entropy values for each brain region.

\begin{tabular}{|c|c|c|}
\hline Region & AD patients & Control subjects \\
\hline Local anterior left & $0.6860 \pm 0.1708$ & $0.6917 \pm 0.0913$ \\
\hline Local anterior right & $0.6671 \pm 0.1560$ & $0.7394 \pm 0.1229$ \\
\hline Local posterior left & $0.5880 \pm 0.1357$ & $0.6540 \pm 0.0890$ \\
\hline Local posterior right & $0.6374 \pm 0.1550$ & $0.6815 \pm 0.1382$ \\
\hline Distant left & $1.0022 \pm 0.1499$ & $1.1136 \pm 0.1925$ \\
\hline Distant right & $0.9994 \pm 0.1630$ & $1.1374 \pm 0.1498$ \\
\hline Interhemispheric & $0.9159 \pm 0.1518$ & $0.9606 \pm 0.0716$ \\
\hline
\end{tabular}

Table 2. $p$-values for the one-way ANOVA tests for each brain region.

\begin{tabular}{|c|c|}
\hline Region & p-value \\
\hline Local anterior left & 0.9220 \\
\hline Local anterior right & 0.2332 \\
\hline Local posterior left & 0.1869 \\
\hline Local posterior right & 0.4801 \\
\hline Distant left & 0.1345 \\
\hline Distant right & 0.0473 \\
\hline Interhemispheric & 0.3838 \\
\hline
\end{tabular}

the characterisation of AD. Specifically, we wanted to test the hypothesis that information content - quantified with KL entropy - between cortical regions in AD patients would be significantly different than in control subjects.

$\mathrm{KL}$ entropy values were lower in $\mathrm{AD}$ patients than in control subjects for the seven brain regions defined in this study. Furthermore, the reduced KL entropy values in AD patients were more apparent for the distant electrodes than for local combination of electrodes, although differences in local information content were also found. The significant reduction in KL entropy values between pairs of distant electrodes might be reflecting the functional impairment in the long cortico-cortical fibre pathways in AD subjects reported by Locatelli et al. [12]. In addition, KL entropy values were lower for closer electrodes (local anterior and local posterior) than for distant or interhemispheric electrode pairs. As KL entropy measures the degree of similarity between two probability distributions, it is logical to assume that the distant or interhemispheric electrodes pairs would be less similar than those in local anterior or local posterior pairs: the smaller the KL entropy values, the more similar the distribution of the two variables.

Significant differences in information transmission in $\mathrm{AD}$ patients EEGs for the distant and interhemispheric electrode pairs in AD patients have been reported using cross mutual information [13]. Although KL entropy is quite different to mutual information, which provides measures of information flow, the similar changes observed with both techniques suggest that the changes in power spectrum observed with KL entropy may also relate to changes in connectivity.

It has been suggested that one of the characteristics of $\mathrm{AD}$ is neocortical disconnection. Cognitive decline comes 
as a result of structural and functional disruption of long cortico-cortical tracts [14]. Moreover, amyloid plaques and neurofibrillary tangles inside nerve cells, two hallmarks of $\mathrm{AD}$, also involve the origins and terminations of long cortico-cortical fibres [15]-[17]. However, KL entropy is a statistical metric quantifying information similarity between two time series. Thus, KL entropy might not only be reflecting changes in axonal connection or cortico-cortical communication in the brain due to $\mathrm{AD}$. The differences between cortical regions in $\mathrm{AD}$ could be due to different factors, like neuronal death, a general effect of neurotransmitter deficiency and loss of connectivity of local neural networks [2].

Although our results indicate that KL entropy could be useful to help in $\mathrm{AD}$ diagnosis, some limitations must be considered. Firstly, the sample size was small. To prove its usefulness as an AD diagnostic tool, this approach should be extended on a much larger patient population. Moreover, the detected changes in the EEG information content between regions might not be specific to AD. Further work must be carried out to examine KL entropy of background EEG activity in other types of dementia to help in the differential diagnosis of AD.

\section{Conclusion}

This pilot study shows that KL entropy might be a useful tool to characterise differences in information content in brain electrical activity in AD patients. Results show that KL entropy values are lower in $\mathrm{AD}$ patients than in agematched control subjects, although not all differences were statistically significant, and are in agreement with the functional impairment in the long cortico-cortical fibre pathways in $\mathrm{AD}$. However, KL entropy measures the degree of similarity between two distributions and might not only be reflecting a possible disruption of corticocortical communication in AD. Further studies with larger sample sizes and in other types of dementia are needed to address the possible usefulness of this methodology in the early detection of AD.

\section{References}

[1] T.D. Bird, Alzheimer's disease and other primary dementias. In E. Braunwald A.S. Fauci, D.L. Kasper, S.L. Hauser, D.L. Longo, \& J.L. Jameson, eds. Harrison's Principles of Internal Medicine (New York: The McGraw-Hill Companies Inc, 2001, 2391-2399).

[2] J. Jeong, EEG dynamics in patients with Alzheimer's disease, Clinical Neurophysiology, 115, 2004, 1490-1505.

[3] D. J. Selkoe, Cell biology of the amyloid betaprotein precursor and the mechanism of Alzheimer's disease, Annual Review of Cell and Developmental Biology, 10, 1994, 373-403.
[4] O.N. Markand, Organic brain syndromes and dementias. In D.D. Daly \& T. A. Pedley, eds. Current Practice of Clinical Electroencephalography (New York, NY: Raven Press Ltd., 1990, 401-423).

[5] R.G. Andrzejak, K. Lehnertz, F. Moormann, C. Rieke, P. David, \& C.E. Elger, Indications of nonlinear deterministic and finite-dimensional structures in time series of brain electrical activity: Dependence on recording region and brain state, Physical Review. E, 64, 2001, p. 061907.

[6] C.E. Shannon, A mathematical theory of communication, Bell Systems Technical Journal, 27, 1948, 379-423 and 623-656.

[7] R. Quian Quiroga, J. Arnhold, K. Lehnertz, \& P. Grassberger, Kullback-Leibler and renormalized entropies: Applications to electroencephalograms of epilepsy patients, Physical Review E, 62(6), 2000, 83808386.

[8] R. Gray, Entropy and Information Theory (New York, Springer-Verlag, 1990).

[9] S. Guiasu, Information Theory with Applications (New York, McGraw-Hill, 1977).

[10] M.F. Folstein, S.E. Folstein, \& P.R. McHugh, Mini-mental state. A practical method for grading the cognitive state of patients for the clinician, Journal of Psychiatry Research, 12, 1975, 189-198.

[11] I.A. Rezek \& S.J. Roberts, Stochastic complexity measures for physiological signal analysis, IEEE Transactions on Biomedical Engineering, 45, 1998 11861191.

[12] T. Locatelli, M. Cursi, D. Liberati, M. Franceschi, \& G. Comi. EEG coherence in Alzheimers disease. Electroencephalography and Clinical Neurophysiology, 106, 1998, 229-237.

[13] J. Jeong, J.C. Gore, \& B.S. Peterson, Mutual information analysis of the EEG in patients with Alzheimer's disease, Clinical Neurophysiology, 112, 2001, 827-835.

[14] A.F. Leuchter, T.F. Newton, I.A. Cook, D.O. Walter, S. Rosenberg-Thomson, \& P.A. Lachenbruch, Changes in brain functional connectivity in Alzheimertype and multi-infarct dementia, Brain, 115, 1992, 15431561.

[15] R.C.A. Pearson, M.M. Esiri, R.W. Hiorns G.K. Wilcock \& T.P.S Powell, Anatomical correlates of the distribution of the pathological changes in the neocortex in Alzheimer's disease, Proceedings of the National Academy of Sciences USA, 82, 1985, 4531-4534.

[16] D.A. Lewis, M.J. Campbell, R.D. Terry, \& J.H. Morrison, Laminar and regional distributions of neurofibrillary tangles and neuritic plaques in Alzheimer's disease: a quantitative study of visual and auditory cortices, The Journal of Neuroscience, 7, 1987, 1799-1808.

[17] J. Rogers \& J.H. Morrison, Quantitative morphology and regional laminar distributions of senile plaques in Alzheimer's disease, The Journal of Neuroscience, 5, 1985, 2801-2808. 